\title{
1 Visualization of SpoVAEa protein dynamics in dormant spores of \\ 2 Bacillus cereus and dynamic changes in their germinosomes and \\ 3 SpoVAEa during germination
}

4 Yan Wang ${ }^{1}$, Norbert O. E. Vischer ${ }^{1}$, Demi Wekking ${ }^{1}$, Alessandra Boggian ${ }^{1}$, Peter Setlow ${ }^{2}$, and Stanley Brul ${ }^{1, *}$

$5{ }^{1}$ Molecular Biology and Microbial Food Safety, Swammerdam Institute for Life Sciences, University of Amsterdam, Science

6 Park 904, 1098 XH Amsterdam, The Netherlands; y.wang5@uva.nl (Y.W.); norbertvischer@gmail.com (N.O.E.V.);

7 demi_wekking@outlook.com (D.W.); boggian1alessandra@gmail.com (A.B.)

$8 \quad{ }^{2}$ Department of Molecular Biology and Biophysics, UConn Health, Farmington, CT 06030-3305, USA; setlow@nso2.uchc.edu

$9 \quad$ * Correspondence: s.brul@uva.nl

\section{Abstract}

11 Bacillus cereus spores, like most Bacillus spores, can survive for years depending on their specific

12 structure, and germinate when their surroundings become suitable. Spore germination proteins play

13 an important role in the initiation of germination. Because germinated spores lose the extreme

14 resistance of the dormant state, more information related to the function of germination proteins could

15 be useful to develop new strategies to control B. cereus spores. Prior work has shown that: i) the

16 channel protein SpoVAEa exhibits high frequency movement in the outer leaflet of the inner

17 membrane (IM) in dormant spores of $B$. subtilis; ii) the dynamics of germinosome formation in

18 developing spores of $B$. cereus indicate that the formation of germinosome foci is slower than foci

19 formation of germinant receptor GerR and scaffold protein GerD. However, the dynamics of movement of SpoVAEa in $B$. cereus spores, and the complete behavior of the germinosome in germinated spores of $B$. cereus are still unclear. In this study, we found that the SpoVAEa fluorescent foci in dormant spores of $B$. cereus redistribute at a lower frequency than in $B$. subtilis, and likely colocalize with GerD in dormant spores. Our results further indicate that: i) overexpression of $\operatorname{GerR}(\mathrm{A}$ C-B)-SGFP2 and SpoVAEa-SGFP2 with GerD-mScarlet-I from a plasmid leads to more heterogeneity and lower efficiency of spore germination in B. cereus; ii), germinosome foci composed of GerR(A-CB)-SGFP2 and GerD-mScarlet-I were lost prior to the phase transition in germination; and iii) GerDmScarlet-I foci spread out but continued to exist beyond the phase transition of $B$. cereus spores.

Keywords: Bacillus cereus, spores, SpoVAEa, GerR, GerD, germination 


\section{2 \\ Introduction}

33 Bacillus cereus is a Gram-positive, rod-shaped, spore forming bacterium found in soil. The vegetative 34 cells of $B$. cereus can form endospores under harsh environmental conditions, and spores are 35 capable of surviving for years due to spore-specific features [1]. These specific properties also lead to major challenges to food safety once $B$. cereus contaminates foods, for example dairy products, rice, and chilled foods $[2,3]$. The major specific structural features of spores include the spore core containing chromosomal DNA, the inner membrane (IM) where germinant receptors (GRs) are located along with the germinosome scaffold protein GerD and channels for CaDPA, the germ cell wall with a thin peptidoglycan layer, the cortex with a thick peptidoglycan layer, the outer membrane, the proteinaceous coat, and finally the exosporium in some Bacillus species, including B. cereus [4].

Dormant spores can initiate germination and outgrow into vegetative cells when GRs sense nutrients in the environment, such as amino acids, inosine, and sugars. Additionally, previous work indicates that GerD acts as a scaffold protein in localizing GRs in the $B$. subtilis spore IM [5,6], and the $B$. cereus GerR GR has also been shown to interact with GerD in the dormant spore IM [7]. Another important group of spore germination proteins are the SpoVA proteins encoded by the spoVA operon which constitute a CaDPA channel in the IM of bacterial spores. The SpoVA proteins in spores of $B$. subtilis are SpoVAA, SpoVAB, SpoVAC, SpoVAD, SpoVAEb, SpoVAEa, and SpoVAF and are made only in developing spores [8]. The SpoVA proteins in spores of $B$. cereus are SpoVAA (BC_4070), SpoVAB (BC_4069), SpoVAC (BC_4068), SpoVAD (BC_4067), SpoVAEb (BC_4066), SpoVAEa (BC_4065) and SpoVAF (BC_4064) encoded in one operon, and SpoVAC (BC_5147), SpoVAD (BC_5148), and SpoVAE (BC_5149) in another operon. Previous work suggests that SpoVAEa of $B$. subtilis spores, a soluble protein on the outer surface of the spore $\mathrm{IM}$, plays a role in the communication with the germinant binding GRs. It is thus possible that SpoVAEa of $B$. subtilis spores could stimulate the opening of the SpoVA channel, thereby allowing CaDPA release $[9,10]$. Recent work in our lab has shown that $B$. subtilis SpoVAEa fused to GFP and expressed on the chromosome in dormant spores exhibits random high frequency movement in the spore IM [11]. However, there is only a limited understanding of the location and physical state of SpoVAEa in dormant spores of the food pathogen B. cereus, nor whether SpoVAEa and GerD proteins colocalize at least transiently.

The spore germination process in Bacilli and Clostridia has been reviewed in the past years [12-14]. Initially germinants bind to GRs, followed by large-scale release of monovalent cations and then CaDPA release mediated by the SpoVA proteins $[15,16]$. The kinetics and heterogeneity of spore germination triggered by L-alanine have been analyzed giving the frequency distribution at both the population level and in individual spores of $B$. cereus strain $T$ using phase-contrast and fluorescence microscopy [17-19]. Previous work suggests that GerR(A-C-B) is localized in the spore IM using fluorescent reporter protein fusions and the membrane dye FM 4-64, and is primarily responsible for germinosome, GerR(A-C-B) and GerD could be visualized using fluorescent reporter proteins and this 
70 formation of GerR(A-C-B)-SGFP2 and GerD-mScarlet-I foci, with significant heterogeneity in

71 formation of germinosomes foci [7]. However, little information is known about the overall changes or

72 behavior of germination proteins during the germination process of $B$. cereus spores.

73 The strongly enhanced green fluorescent protein (SGFP2) and mScarlet-I have been successfully 74 used in the visualization of spore germination proteins GerR(A-C-B) and GerD in spores of $B$. cereus

75 ATCC 14579 using low-copy number plasmid expression vectors [7,20]. In this work, we aimed to 76 visualize the movement of SpoVAEa with SGFP2 in dormant spores of B. cereus ATCC 14579 using 77 fluorescence microscopy, and to analyze the fluorescence distribution by the changes, either up or 78 down, in the full width at half maximum (FWHM) of the fluorescence. Additionally, the phase contrast 79 intensity and fluorescence changes of germinosome foci formed by GerR(A-C-B)-SGFP2 and GerD80 mScarlet-I, and SpoVAEa-SGFP2 and GerD-mScarlet-I were tracked by a time-lapse microscope 81 equipped with phase-contrast and fluorescence analysis options. In the present study, we found that 82 SpoVAEa-SGFP2 foci exhibited random movements in the IM, and likely colocalized with GerD$83 \mathrm{mScarlet-I}$ in dormant spores of $B$. cereus. The results also suggested that expression of GerR(A-C-B) 84 and SpoVAEa proteins with GerD affected germination efficiency and led to slower and more 85 heterogeneous spore germination in $B$. cereus. Upon addition of germinant, germinosome FRET foci 86 were lost and GerR(A-C-B)-SGFP2 as well as GerD-mScarlet-I germinosome foci decreased 87 significantly in intensity. Yet some GerR(A-C-B)-SGFP2 foci and GerD-mScarlet-I foci continued to exist and apparently colocalize also after phase transition, suggesting that germinosome complexes may persist beyond the germination event. Finally, loss of SpoVAEa-SGFP2 foci occurred upon phase transition, while spread of GerD-mScarlet-I foci continued beyond phase-transition.

\section{Results}

\section{Movement of SpoVAEa foci in dormant spore of B. cereus}

Previous studies showed that full width at half maximum (FWHM) can be used to quantitate fluorescence distribution of spore proteins [11]. In this study, we used dormant spores of $B$. cereus strain 014 expressing the fusion protein SpoVAEa-SGFP2 from a plasmid to observe the movement of SpoVAEa-SGFP2 foci. The percent changes of FWHM in 100 frames of an individual B. cereus spore during $5 \mathrm{sec}$ was presented as up (positive percentage) or down (negative percentage) compared to the first frame. It was noted that there were different random movements in $B$. cereus spores 1, 2, 3 and 4 (Figure 1.A). The percent changes of FWHM in B. subtilis exhibited a wider boundary and higher frequency changes, either up or down, compared to $B$. cereus spore 2 (Figure 1.B). This result suggested that the SpoVAEa-SGFP2 foci in spores of $B$. cereus and $B$. subtilis both moved, but behaved somewhat differently. In particular, SpoVAEa fluorescent foci of $B$. subtilis spores redistribute at a higher frequency than those of $B$. cereus. This difference may be caused by the fact that we look at different species with a different protein complement and with genomic expression in $B$. subtilis versus expression from a plasmid in $B$. cereus. 

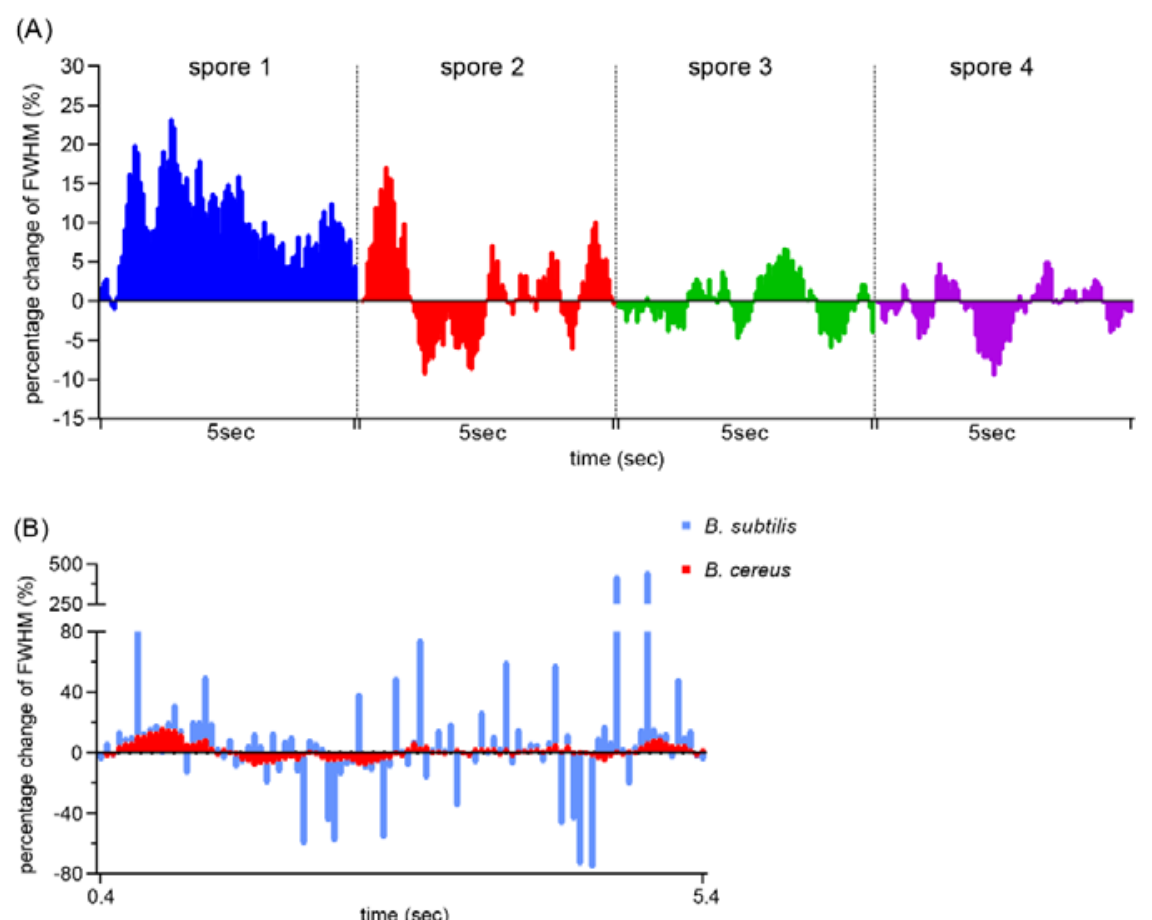

Figure 1. Comparison of the movement of SpoVAEa-SGFP2 foci in dormant spores of $B$. cereus and $B$. subtilis.

Panel $A$, the percent changes of FWHM in individual spores $1,2,3$ and 4 of $B$. cereus. Panel $B$, the percent changes of FWHM in a $B$. cereus spore 2 (red square) and a $B$. subtilis spore (blue square). The positive and negative percentages of columns indicate the up and down of the tendency of fluorescence FWHM distribution.

\section{SpoVAEa-SGFP2 levels are enhanced by GerD expression in recombinant $B$. cereus} spores

113 Our recent work showed that GerR(A-C-B) and GerD foci are present and colocalized in dormant spores of $B$. cereus [20]. The fusion protein SpoVAEa-SGFP2 was expressed alone in spores of $B$. cereus strain 014, and expressed together with GerD-mScarlet-I in spores of strain 015 in this work.

116 The fluorescence intensity of SGFP2 in strains 014 and 015 are as expected both higher than those 117 of wild type $B$. cereus cells without recombinant protein. However, it is notable that the fluorescence in 118 B. cereus strain 015 looks brighter than in strain 014 (Figure 2.A). When the fluorescence level in the spores of recombinant strains 014 and 015 were compared, $53 \%$ of the 014 spores and $43 \%$ of the 015 spores gave a 2 3 fold higher fluorescence levels compared to the wild type. Furthermore, levels of recombinant spores with 3-fold higher fluorescence level than wild type spores were higher in strain 015 spores than 014 spores (Figure 2.B). These results indicate that SpoVAEa-SGFP2 can be successfully expressed from a plasmid in two recombinant $B$. cereus strains 014 and 015 and leads to 1 to 6 fold higher fluorescence levels than the wild-type in the SGFP2 channel. Moreover, when SpoVAEa-SGFP2 and GerD-mScarlet-I are expressed from the same plasmid, GerD possibly contributes to the stability of SpoVAEa, or possibly enhances the expression of SpoVAEa. 
(A)
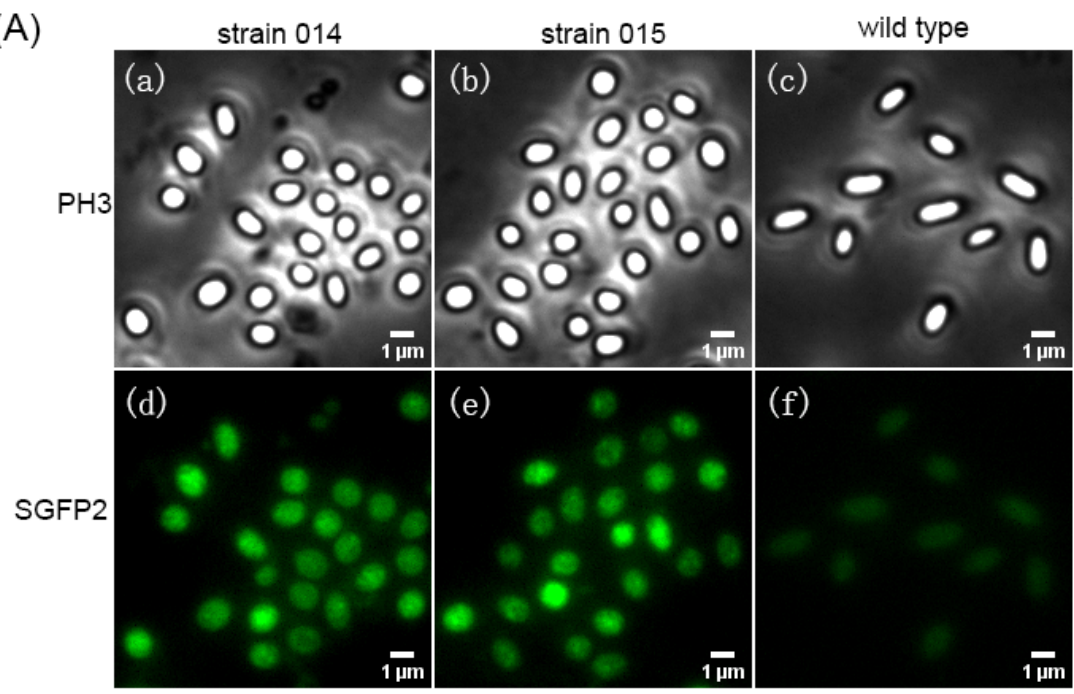

(B)

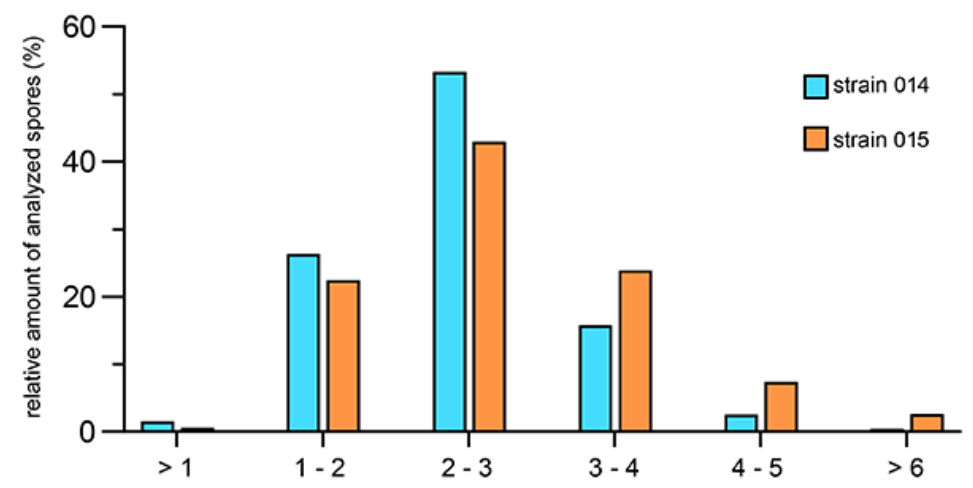

Fluorescence level of recombinant spores compared to wild-type in the SGFP2 image

Figure 2. Visualization and comparison of SpoVAEa-SGFP2 fusion protein fluorescence in dormant spores of $B$. cereus strains 014 and 015 . Panel A, dormant spores of $B$. cereus strains 014,015 and wild type were visualized: (a), (b), and (c) in the PH3 channel, or (d), (e), and (f) in the SGFP2 fluorescence channel. PH3, phase contrast. Panel B, fluorescence level in the SGFP2 channel in spores of strains 014 and 015 compared to wild type. The number of analyzed spores of $B$. cereus strain 014, 015 and wild type were 1564, 2901, and 916, respectively.

\section{Colocalization analysis between SpoVAEa and GerD proteins}

134 The next aim was to study the possible interaction of SpoVAEa-SGFP2 and GerD-mScarlet-I in 135 dormant spores of $B$. cereus. The spectrum of SGFP2 and mScarlet-I have an overlapping region, 136 that may produce a larger Pearson's coefficient, which is a commonly used colocalization indicator, $137[7,22,23]$. To reduce any effects of GerD-mScarlet-I itself, spores of $B$. cereus strain 007 expressing 138 GerD-mScarlet-I was used as a control. The analysis (Fig. 3) showed that the Pearson's coefficient of channels SGFP2 and mScarlet-I in spores of $B$. cereus strain 015 was significantly higher than those

140 in the control. This result indicated that there is likely colocalization between the SpoVAEa-SGFP2 and GerD-mScarlet-I proteins in B. cereus spores. 
(A)
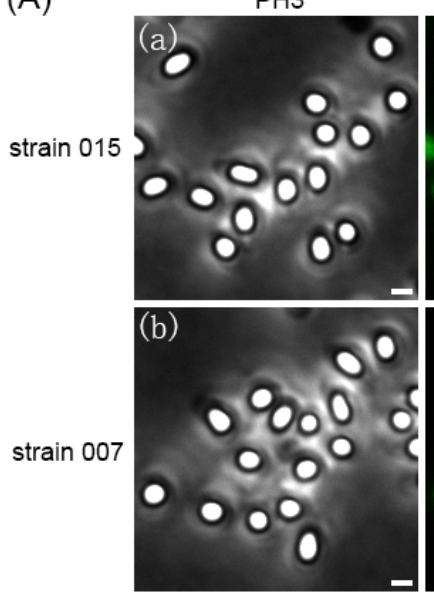

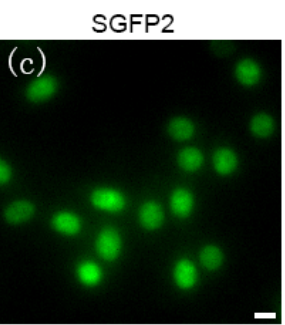

(d)

(B)

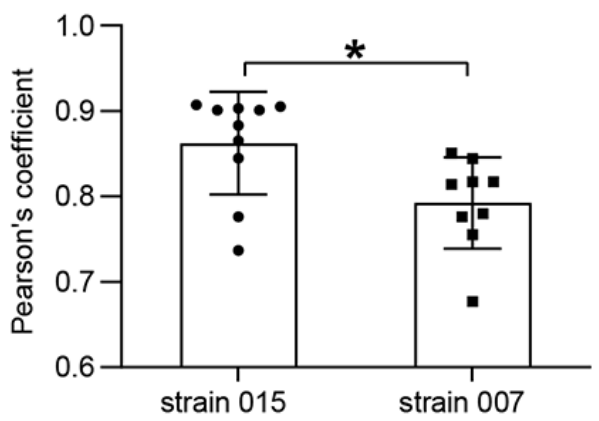

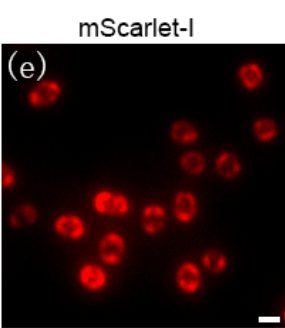

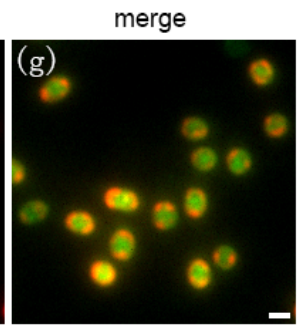

\section{(f)}

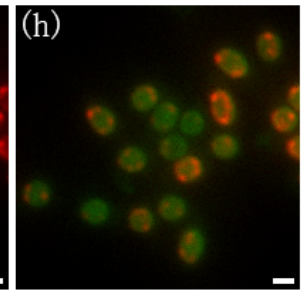

143

144

145

146

147

Figure 3. Co-localization analysis between SpoVAEa and GerD proteins in dormant spores of $B$. cereus strain 015. Panel A, visualization of $B$. cereus strain 015 and 007 spores: (a) and (b) Phase-contrast channel (PH3); (c) and (d), SGFP2 channel; (e) and (f), mScarlet-I channel; (g) and (h), merged image of SGFP2 and mScarlet-I channels. The scale bar is $1 \mu \mathrm{m}$. Panel B, Pearson's coefficient between SGFP2 and mScarlet-I channels. Data are shown as a mean with SD. * $P<0.05$.

\section{Overexpression of GerR and SpoVAEa with GerD affects B. cereus spore germination}

Our recent work suggested that there is a significant kinetic heterogeneity in the formation of a germinosome in developing spores of $B$. cereus [7]. In this study, the initiation time of germination (named germX) is presented as the difference in spores of strain 006 expressing GerR(A-C-B)SGFP2 and strain 014 expressing SpoVAEa-SGFP2 compared to wild type. When GerR(A-C-B)SGFP2 and SpoVAEa-SGFP2 with GerD-mScarlet-I expressed from plasmid in B. cereus strains F06 and 015 respectively, germX exhibited greater heterogeneity than spores of $B$. cereus strains 006 and 014 (Figure 4). In particular, spores of B. cereus strain 007 expressing GerD-mScarlet-I alone from plasmid exhibited a very different germ $X$ of individual spores (Figure 4). Our result indicated that overexpression of GerD-mScarlet-I from plasmid in B. cereus led to more heterogeneity of spore germination.

Previous work has shown that expression of the GerA GR controlled by the strong forespore-specific $s s p B$ promoter in the $B$. subtilis genome can significantly increase the rate of germination triggered by L-alanine [24]. Our results showed that spores of all different $B$. cereus strains started to germinate by 
bioRxiv preprint doi: https://doi.org/10.1101/2022.01.10.475594; this version posted January 10,2022 . The copyright holder for this preprint (which was not certified by peer review) is the author/funder, who has granted bioRxiv a license to display the preprint in perpetuity. It is made available under aCC-BY-NC-ND 4.0 International license.

1621,10 and $>20$ min, namely groups germX_1, germX_10, and germX_>20. Most spores were in group germX_1, with $81.2 \%, 82.9 \%, 48.5 \%, 97.7 \%, 74.7 \%$, and $99.8 \%$ of spores of $B$. cereus strains $\mathrm{F} 06$, 006, 015, 014, 007 and wild type, respectively (Figure 5). Overexpression of GerR(A-C-B) with or without GerD in strains F06 and 006 led to significantly lower germination efficiency compared to wild type spores, with effects in F06 spores slightly greater than in 006 spores. The results also showed that overexpression of SpoVAEa alone in spores of strain 014 had no effect on germination efficiency compared to wild type. However, expression of SpoVAEa with GerD in spores of B. cereus strain 015 significantly $(P<0.0001)$ slowed germination compared to wild type spores (Figure 5$)$. Importantly, whether GerD was expressed alone from a plasmid in B. cereus strain 007 or along with GerR in strain F06 or SpoVAEa in strain 015 , the overexpression of GerD led to a significantly lower germination efficiency compared to wild type spores (Figure 5). Overall, our results indicate that overexpression of the GerR GR and SpoVAEa with GerD from plasmids affects the germination efficiency of $B$. cereus spores.
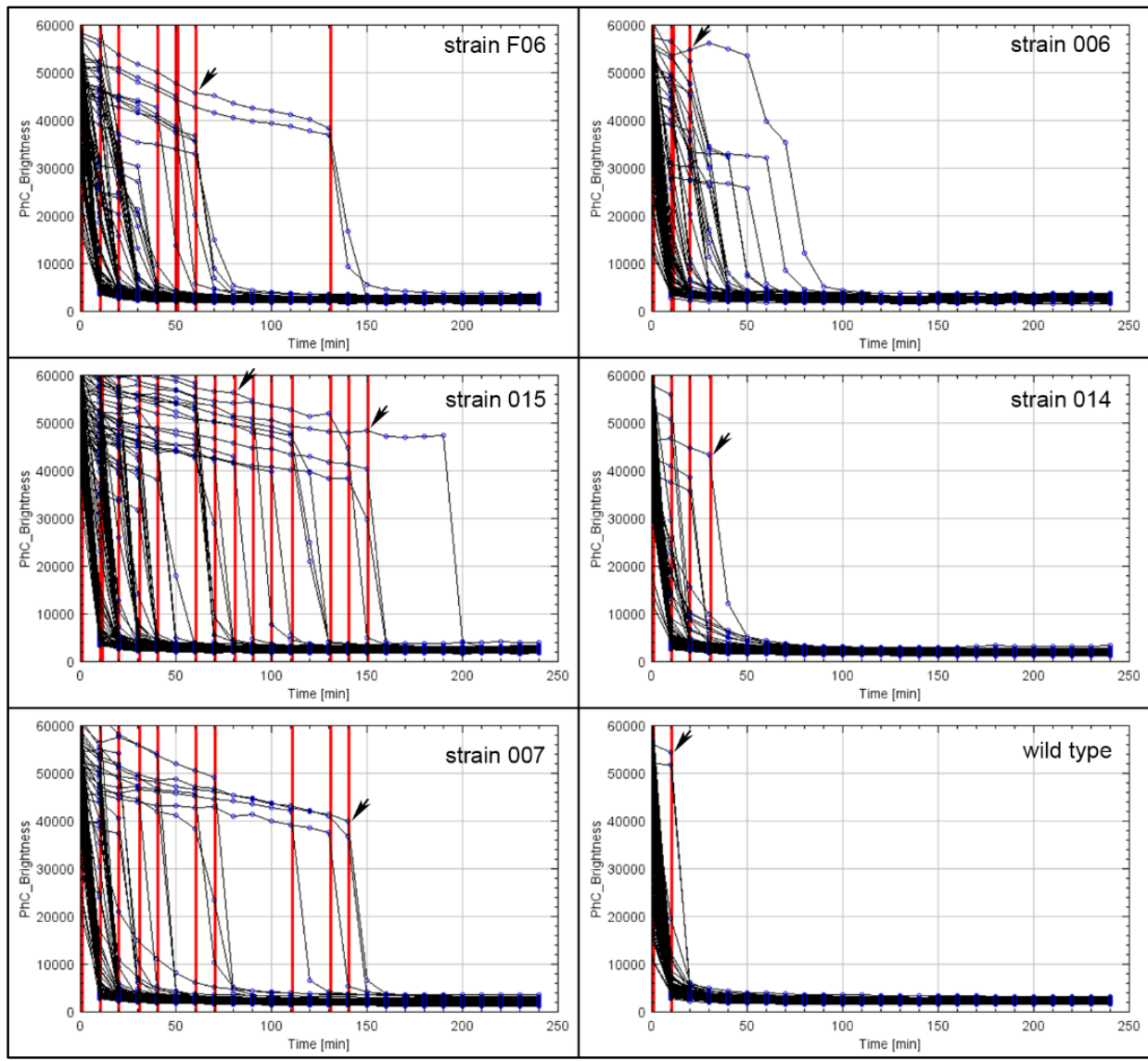

Figure 4. The phase plots show the germinated spores of $B$. cereus strains F06, 006, 015, 014, 007 and wild type. The red lines and black arrows indicate the initiation time of spore germination, termed germX. Each black line indicates the change of phase-contrast intensity in an individual spore during germination. The numbers of analyzed spores of B. cereus strains F06, 006, 015, 014, 007 and wild type were 108, 92, 122, 233, 107 and 265, respectively. 
181

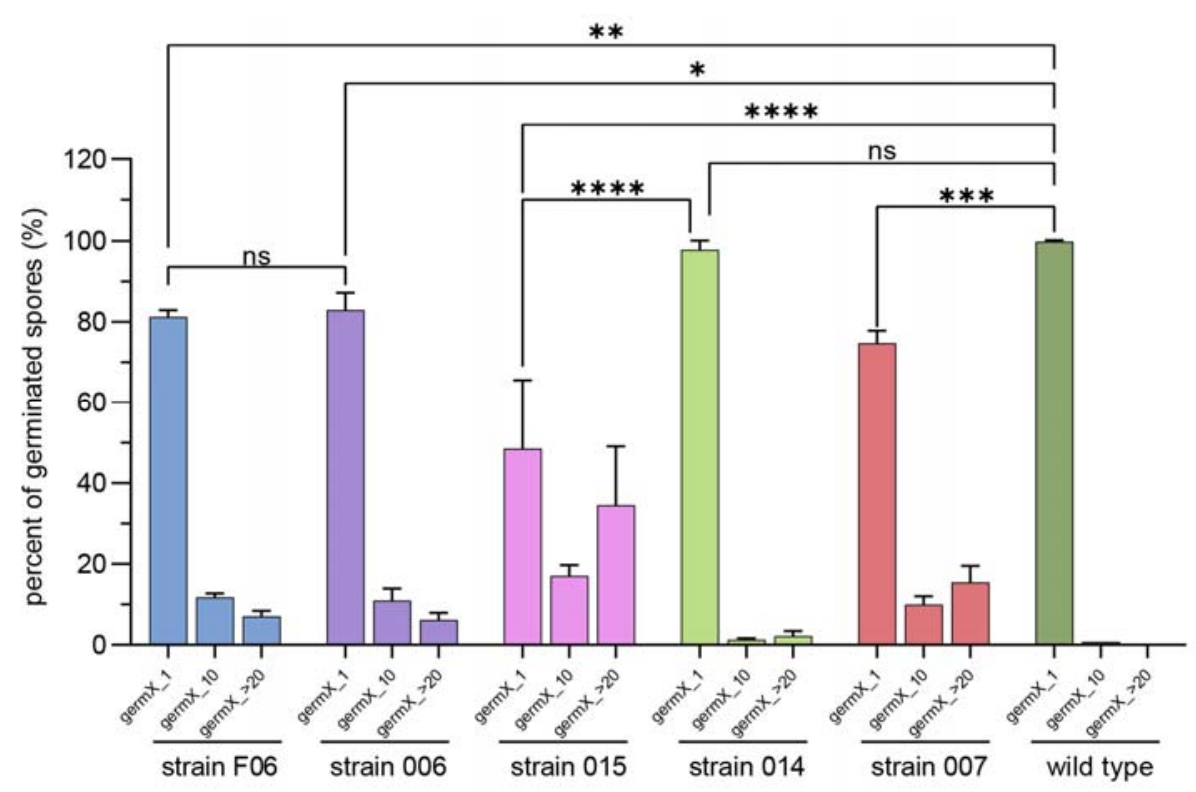

Figure 5. Germination of spores of $B$. cereus strains F06, 006, 015, 014, 007 and wild type were analyzed based on the different germX times: germX_1, initiation time of germination at $\leq 1 \mathrm{~min}$; germX_10, initiation time of germination at <10 min; germX_>20, initiation time of germination more than $20 \mathrm{~min}$. Data are shown as the mean with SD and are averages of three independent experiments. The numbers of analyzed germinated spores of different $B$. cereus strains are listed in Table S2. ns, not significant; ${ }^{*}, P<0.05 ;{ }^{* *}, P<0.01,{ }^{* \star *}, P<0.001$; ${ }^{* \star \star *}$, $P<0.0001$.

\section{Dynamics of germinosome behavior upon germination triggered by L-alanine in $B$. cereus spores}

Our recent study suggested that the formation of FRET foci of GerR(A-C-B)-SGFP2 and GerDmScarlet-I could be significantly slower than the foci in SGFP2 and mScarlet-I channels in B. cereus spores [7]. In this work, we aimed to track the dynamic changes of germinosome FRET foci upon germination triggered by L-alanine in spores of $B$. cereus strain F06 expressing GerR(A-C-B)-SGFP2 and GerD-mScarlet-I fusion proteins from a plasmid. The phase-contrast channel $(\mathrm{PH} 3)$ recorded the transition between a phase-bright individual spore to a phase-dark spore at $1 \mathrm{~min}$ and $10 \mathrm{~min}$ of the germination time in groups germX_1 and germX_10, respectively (Figure 6.A, Table S3). Upon the phase transition in the germX_1 group, the intensity of germinosome FRET foci at 10 min was significantly lower than at the beginning (0 min), as well as that of GerR(A-C-B)-SGFP2 and GerDmScarlet-I foci (Figure 6.B, Table S3). In addition, the intensity of the FRET foci of germX_10 spores at $30 \mathrm{~min}$ was significantly lower compared to $20 \mathrm{~min}$, that is, the intensity drop occurred always after phase transition (Figure 6.B, Table S3). In addition, any remaining GerD-mScarlet-I foci became diffuse in germinated spores of groups germX_1 and germX_10 (Figure 6.B). These results indicate that the germinosome FRET foci in spores of $B$. cereus were largely lost shortly after the phase transition. Some GerR(A-C-B)-SGFP2 foci and GerD-mScarlet-I foci continued to exist and apparently colocalize also after phase transition, suggesting that germinosome complexes may persist beyond the germination event. 


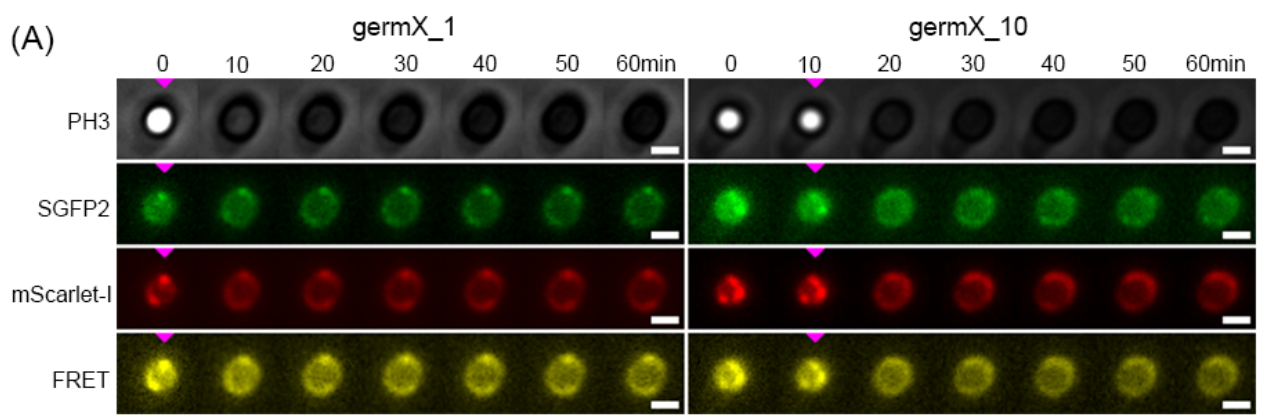

(B)

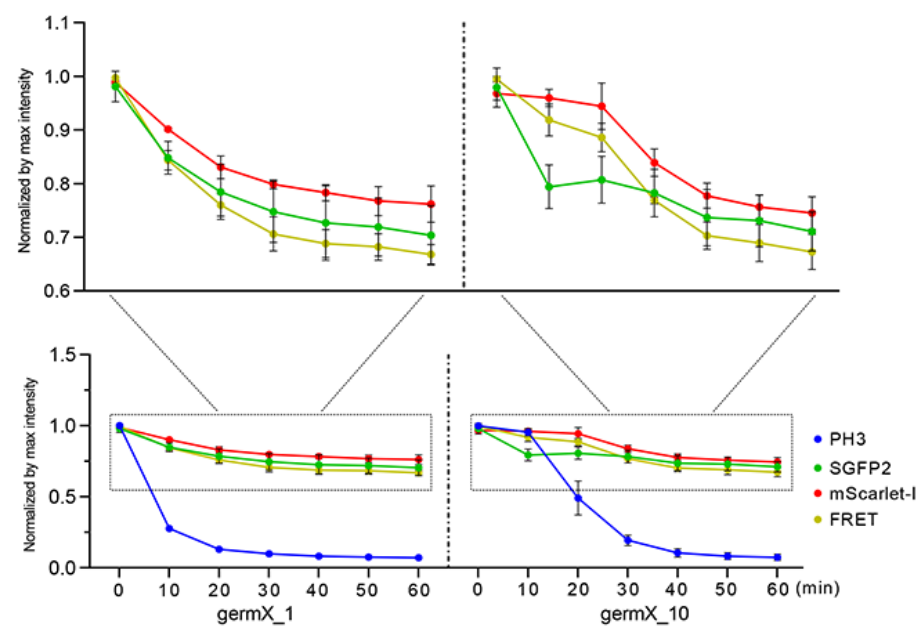

Figure 6. Dynamic changes in germinosome foci upon germination of $B$. cereus strain F06 spores. Panel A, visualization of the changes oinGeR-SGFP2, GerD-mScarlet-I and germinosome foci at $10 \mathrm{~min}$ intervals over 60 min. The left column is PH3, SGFP2, mScarlet-I and FRET channels of group germX_1. The right column is PH3, SGFP2, mScarlet-I and FRET channels of the germX_10 group. PH3, phase-contrast. The pink triangles indicate the initiation of germination. The scale bar is $1 \mu \mathrm{m}$. Panel B, the line charts of PH3, SGFP2, mScarlet-I and FRET channels. Left column, germX_1 group; right column, germX_10 group. Data are shown as the mean with SD and are averages of three independent experiments. The number of analyzed germinated spores of $B$. cereus strains F06 is listed in Table S2. The statistical analysis of the later time points in line charts in comparation to the previous one are listed in Table S3. triggered by L-alanine

In this work, recombinant spores of $B$. cereus strain 015 expressing SpoVAEa-SGFP2 and GerDmScarlet-I fusion proteins from a plasmid were used to visualize the dynamic changes of SpoVAEa and GerD upon germination initiated by L-alanine. The results showed that the phase-contrast intensity of germinated spores at $10 \mathrm{~min}$ in group germX_1 was very greatly decreased compared to that of phase-bright spores at $0 \mathrm{~min}$. In the group germX_10, the phase transition occurred and the phase-contrast intensity of phase-dark germinated spores at 20 min was greatly decreased compared to that at $10 \mathrm{~min}$ (Figure 7, Table S4). The fluorescence intensity in the SGFP2 or mScarlet-I channel of germinated spores at $10 \mathrm{~min}$ in group germX_1 was decreased compared to that of phase-bright spores at $0 \mathrm{~min}$, but this reduction was not significant $(P>0.05)$. The results showed that the 


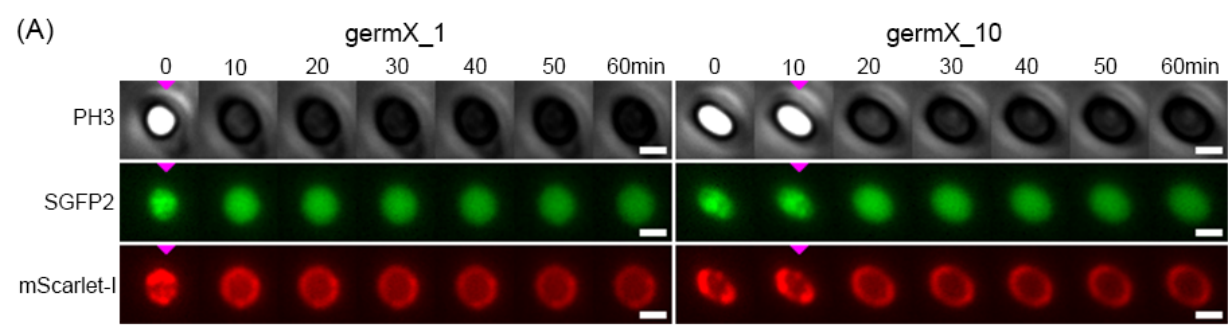

(B)

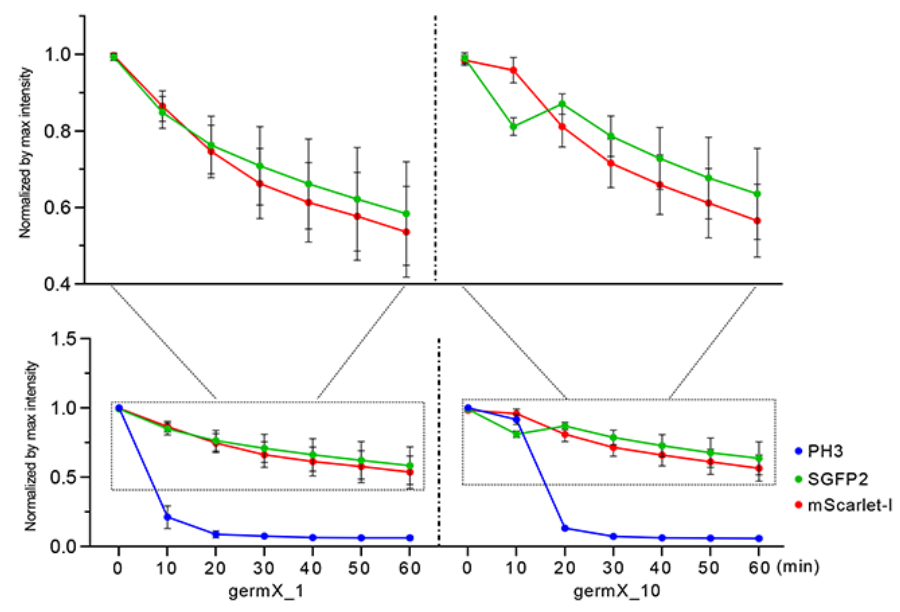

Figure 7. Dynamic changes in SpoVAEa-SGFP2 and GerD-mScarlet-I during germination of $B$. cereus strain 015 spores. Panel A, visualization of changes in SpoVAEa-SGFP2 and GerD-mScarlet-I at 10 min intervals over 60 min. The left column is PH3, SGFP2 and mScarlet-I channels of group germX_1. The right column is PH3, SGFP2 and mScarlet-I channels of the germX_10 group. PH3, phase-contrast. The pink triangles indicate the initiation of germination. The scale bar is $1 \mu \mathrm{m}$. Panel B, the line charts of PH3, SGFP2 and mScarlet-I channels. Left column, germX_1 group; right column, germX_10 group. Data are shown as the mean with SD and represent three independent experiments. The numbers of analyzed germinated spore of $B$. cereus strains 015 are listed in Table S2. The statistical analysis of the latter time points in line charts in comparation to the previous one is listed in Table S4.

\section{Discussion}

B. cereus spores, like most Bacillus spores, have various resistance characteristics due to spore specific structures, and can restart metabolism only after spore germination has been completed. The nutrient germination of spores is initiated by germinant binding to specific GRs localized in spore's IM, including GerR, GerK, GerG, GerL, GerQ, Gerl, and GerS in spores of B. cereus, with GerR triggering germination with L-alanine [21,25]. In addition, SpoVAEa is an IM component of the SpoVA protein CaDPA channel and GerD is a scaffold protein playing an important role in germinosome formation and thus spore germination in $B$. subtilis and $B$. cereus $[6,7,9]$. To extend these latter observations, 
251 we have now studied the dynamic changes of SpoVAEa protein in dormant and germinated spores of

252 B. cereus, and the kinetic changes in germinosome foci during the germination process.

253 Based on previous observations, the expression level of GerD and SpoVA proteins in B. subtilis 254 spores are $\sim 10^{2}$-fold and $10^{3}$-fold higher than those of GRs [26]. Fluorescence microscopy of $B$. 255 cereus spores showed clear foci of GerD-mScarlet-I and indistinct foci of SpoVAEa-SGFP2, indicating 256 that a possible reason could be the different level of the two proteins. However, the weaker 257 fluorescent signal of SpoVAEa-SGFP2 than that of GerD-mScarlet-I in our work might be caused by 258 expression of only one subunit of the SpoVA complex, or perhaps SpoVAEa has a lower expression 259 level than other SpoVA proteins [9].

260 In this study, recombinant B. cereus spores overexpressing $\operatorname{GerR}(\mathrm{A}-\mathrm{C}-\mathrm{B})$ and SpoVAEa with and without GerD from a plasmid were used to assess the effects of these proteins on germination triggered by L-alanine. Spores expressing GerR(A-C-B) with GerD in group germX_1 exhibited two significant reductions in germination, 0 min versus $10 \mathrm{~min}$ and $10 \mathrm{~min}$ versus $20 \mathrm{~min}$ in the phasecontrast channel, but there was only one significant reduction 0 min versus 10 min with spores expressing GerR(A-C-B) alone (Figure S1, Table S3, Table S5); note similar results found in group germX_10. However, spores overexpressing SpoVAEa with and without GerD both presented only one significant reduction in the phase-contrast channel in groups germX_1 and germX_10 (Figure S1, Table S4, Table S5). These results indicated that overexpression of GerD has an inhibitory effect on spore germination heterogeneity and efficiency, and note that the heterogeneity of spore germination is consistent with previous work [17,19]. Considering initiation of spore nutrient germination, a recent study suggested that in $B$. subtilis, GerAB is responsible for sensing L-alanine, indicating that the function of GerA's B subunit is to detect nutrient germinants [27]. Our recent studies also suggest that there is interaction of GerRB with GerD and with a GR B subunit possibly bound by L-alanine $[7,28]$. Finally, the possible reason for this inhibitory effect can be that GerD might occupy the limited binding site or position on the surface of GerRB when spore germination is triggered by L-alanine.

Our recent results using FRET-based analysis indicate that there is interaction between GerR(A-C-B)SGFP2 and GerD-mScarlet-I, moreover, the dynamics of germinosome formation suggest that the formation of foci in the FRET channel could be significantly slower than formation of the GerR(A-C-B)SGFP2 and GerD-mScarlet-I foci. To further assess this interaction, we observed the changes of germinosome foci upon germination initiated by $L$-alanine in $B$. cereus spores. In this experiment, the protein FRET pairs, GerR(A-C-B)-SGFP2 and GerD-mScarlet-I, were expressed from a plasmid and driven by their native promoters during sporulation. Possibly consistent with the role of the $B$ subunit of GerA in $B$. subtilis, GerRB may also be responsible for initiating germination with L-alanine in $B$. cereus (Figure 6). Once the process of spore germination was initiated, our results showed that some GerD SpoVAEa colocalization likely occurs and germinosome FRET positive foci were lost upon phase transition while some germinosome complexes may continue to exist beyond this time point. Figure 8 shows a hypothetical sequence of events that may occur during spore germination. A note of caution is warranted because the germination proteins were overexpressed from a plasmid and 
289

290

291

292

293

294

295

296

297

298

299

300

301

302

303

304

305

306

307

308

309

310

311

312

313

314

315

316

317

318

evidently may disrupt the dynamic balance in germination protein assembly in sporulation and germination. Importantly though, all germination proteins studied were expressed from the plasmid under the control of their respective native promoters allowing relative expression differences to be conserved.

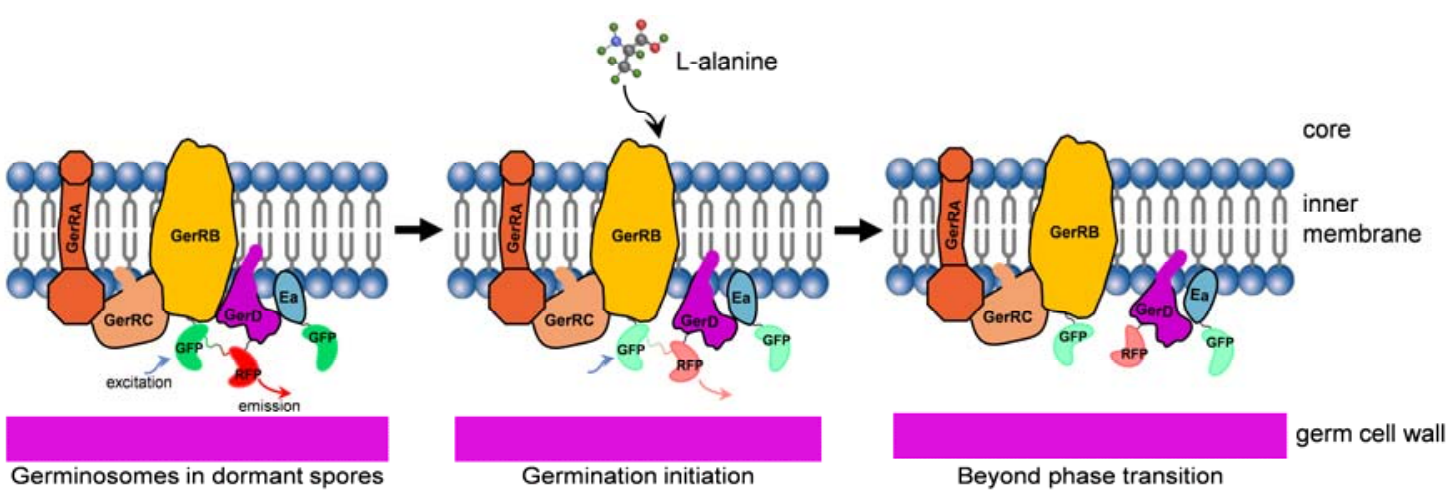

Figure 8. The proposed model of germinosome dynamics during germination triggered by L-alanine in $B$. cereus spores. The left most panel: i) FRET positive germinosome formation due to FRET interaction between GerR(AC-B)-SGFP2 and GerD-mScarlet-I; the darker green-red line between GFP (deep green) and RFP (deep red) indicates the energy transfer path in the FRET event between GerR(A-C-B) and GerD; ii) likely colocalization between SpoVAEa and GerD proteins, albeit transient; GFP - SGFP2; RFP - mScarlet-I; Ea - SpoVAEa. The middle panel: i) FRET positive germinosomes are largely lost upon the phase transition in germination initiation caused by L-alanine; the FRET signal (light green-red line) between GFP (light green) and RFP (light red) has become weak, indicating that FRET events between GerR(A-C-B) and GerD have been gradually lost, consistent with GerD and GerRB-GFP moving apart; ii) the SpoVAEa-SGFP2 and GerD-mScarlet-I fluorescence intensities have decreased upon initiation of germination. The right most panel: i) there are no longer any FRET positive germinosomes, but some GerR(A-C-B)-SGFP2 foci (light green) and GerD-mScarlet-I foci (light red) continue to exist, indicating colocalization of GerR(A-C-B) and GerD after the phase transition; ii) some GerD foci also continue to exist and likely colocalize, perhaps transiently with SpoVAEa.

In summary, the SpoVAEa-SGFP2 protein exhibits random movement on the outer surface of spores' IM and a likely transient co-localization with GerD-mScarlet-I in dormant spores of $B$. cereus. Studying spore germination in phase-contrast microscopy suggested that overexpression of GerR(A-C-B)SGFP2 and SpoVAEa-SGFP2 with GerD-mScarlet-I from a plasmid leads to more heterogeneity and lower efficiency of spore germination in $B$. cereus pointing to the need for future studies to investigate the stoichiometry of the germinosome components in $B$. cereus in more detail. The dynamics of germination showed that germinosome foci composed of GerR(A-C-B)-SGFP2 and GerD-mScarlet-I were lost soon after the phase transition. Further work related to the machinery of spore germination, should likely focus on detailed interaction studies between the SpoVA channel and GerD, or the SpoVA channel and other GRs using site-directed mutagenesis guided by molecular dynamics protein modelling.

\section{Materials and Methods}




\section{Recombinant plasmids and $B$. cereus strains}

320 The recombinant plasmids and $B$. cereus strains used in this study are listed in Table 1. All primers 321 used are listed in Table S1. The recombinant plasmids were constructed as described in our previous 322 studies [7,20]. Briefly, the region of $226 \mathrm{bp}$ located in the upstream region of the spoVA operon was 323 considered as the promoter region of gene spoVAEa and named PEa. The PEa fragment was 324 inserted into pHT315 between $K p n I$ and $X$ ba I sites resulting in plasmid pHT315-PEa. Next, the 325 spoVAEa (BC_4065) gene was amplified from genomic DNA of B. cereus ATCC 14579 (GenBank: 326 AE016877) using a pair of primers, 315_YW-42 and 315_YW-43. The SGFP2 gene with stop codons 327 was fused to the $3^{\prime}$ end of the spoVAEa gene using a two-fusion PCR. The fusion product was 328 inserted into pHT315-PEa between Xba I and Hind III sites. The resulted ligation product was 329 transformed into competent E. coli cells and selection of positive clones giving plasmid pHT315-f14. 330 The fusion fragment PD-gerD-mScarlet-I was amplified from plasmid pHT315-f05 and inserted into 331 pHT315-f14 between Kpn I and EcoR I sites, giving plasmid pHT315-f15. The correct construction of 332 recombinant plasmids $\mathrm{pHT} 315-\mathrm{f} 14$ and $\mathrm{pHT} 315-\mathrm{f} 15$ was confirmed by sequencing, followed by 333 electroporation into competent B. cereus ATCC 14579 cells, and selection and confirmation with 334 colony PCR of an erythromycin-positive single colony.

Table 1. B. cereus strains and plasmids used in this study

\begin{tabular}{|c|c|c|c|}
\hline Strains & Plasmid Present $(+)$ & Description of Inserted Genes & $\begin{array}{l}\text { Sources or } \\
\text { references }\end{array}$ \\
\hline B. cereus ATCC 14579 & wild type & No & Lab stock \\
\hline strain 014 & +pHT315-f14 Ery ${ }^{r}$ & PEa-spoVAEa-SGFP2 & This study \\
\hline strain 015 & +pHT315-f15 Ery ${ }^{r}$ & $\begin{array}{l}\text { PEa-spoVAEa-SGFP2 } \\
\text { and PD-gerD-mScarlet-I }\end{array}$ & This study \\
\hline strain 006 & +pHT315-f01 Ery ${ }^{r}$ & PR-gerR(A-C-B)-SGFP2 & [7] \\
\hline strain 007 & +pHT315-f05 Ery ${ }^{r}$ & PD-gerD-mScarlet-I & [7] \\
\hline strain 010 & +pHT315-f10 Ery ${ }^{r}$ & PD-gerD-SGFP2 & [7] \\
\hline strain F06 & +pHT315-f06 Ery ${ }^{r}$ & $\begin{array}{l}\text { PR-gerR(A-C-B)-SGFP2 } \\
\text { and PD-gerD-mScarlet-I }\end{array}$ & {$[7]$} \\
\hline
\end{tabular}

Abbreviations: PEa, promoter of spoVA operon; PR, promoter of gerR operon; PD, promoter of gerD; Ery' ${ }^{r}$, resistant to 337 erythromycin.

\section{High frequency time-lapse image acquisition and analysis}

339 Dormant spores of $B$. cereus strain 014 were prepared and purified as described in previous work [20]. 340 A Nikon Eclipse Ti-E microscope (Nikon Instruments, Tokyo, Japan) equipped with a sCmos camera 341 (Hamamatsu Flash 4.0 V2, Hamamatsu City, Japan) and wide-field fluorescence components was 342 used to capture 100 frames of 14-bit SGFP2 images (excitation at $488 \mathrm{~nm}$ and emission at $535 \mathrm{~nm}$ ) 343 with an exposure time of $50 \mathrm{~ms}$ for each frame and no delay interval. An individual spore located in 
344100 frames was selected, duplicated, and analyzed by the plugin Adrian's FWHM in ImageJ. The

345 percent change of FWHM in the second frame to the hundredth frame relative to the FWHM in the

346 first frame was calculated, and the graph was made by the software of GraphPad Prism version 9.3.

347 Images of SpoVAEa-SGFP2 expressed in spores of $B$. cereus strains 014 and 015;

\section{8 acquisition and analysis}

349 The preparation of $B$. cereus dormant spores and their visualization were carried out as described in 350 our previous work [20]. Spores of $B$. cereus strain 014 expressing SpoVAEa-SGFP2 and spores of $B$. 351 cereus strain 015 expressing SpoVAEa-SGFP2 and GerD-mScarlet-I were captured in the phase352 contrast and SGFP2 (excitation at $470 \mathrm{~nm}$ and emission at $516 \mathrm{~nm}$ ) channels using a Nikon Eclipse 353 Ti-E microscope. Images were analyzed by the ObjectJ SporeAnalyzer_1c.ojj in Fiji/ImageJ 354 (https://sils.fnwi.uva.nl/bcb/objectj/examples/SporeAnalyzer/MD/SporeAnalyzer.html).

\section{Co-localization assays and data analysis}

356 Dormant spores of $B$. cereus strains 015 and 007 were prepared and purified as described in our 357 previous work [20]. Spores of $B$. cereus strains 015 and 007 were captured in three channels: phase358 contrast, SGFP2 (excitation at $470 \mathrm{~nm}$ and emission at $516 \mathrm{~nm}$ ) and mScarlet-I (excitation at $555 \mathrm{~nm}$ 359 and emission at $593 \mathrm{~nm}$ ) using a Nikon Eclipse Ti-E microscope. All acquired images in the co360 localization assay were processed with ImageJ. The SGFP2 and mScarlet-I images were used to 361 calculate the co-localization indicator Pearson's coefficient by the plugin JACoP in ImageJ [22].

\section{Germination assays by time-lapse imaging and data processing}

363 Dormant spores of $B$. cereus strains F06, 006, 015, 014, 007 and wild-type spores were prepared and 364 purified as described previously [20]. Microscope slides were prepared as described previously [29]. 365 Briefly, a $65 \mu \mathrm{l}$ size Gene frame with $0.25 \mathrm{~mm}$ thickness (Thermofisher Scientific, The Netherlands, 366 Cat. No.: AB0577) was attached on the center of a normal microscope slide. A liquid mixture for an 367 agarose pad was made with a 1:1 mixture of $2 x$ germination buffer (see below) and $2 \%$ agarose in a 368 heat block at $55^{\circ} \mathrm{C} .60 \mu \mathrm{l}$ of the liquid mixture was pipetted on the area of frame, immediately pressed 369 with another slide and placed at $4^{\circ} \mathrm{C}$ for at least $20 \mathrm{~min}$ to solidify.

370 Dormant spores suspended in ice-cold PBS ( $\mathrm{pH}$ 7.4) were heat activated for $15 \mathrm{~min}$ at $70^{\circ} \mathrm{C}$ and 371 washed three times with ice-cold PBS ( $\mathrm{pH} 7.4)$ by centrifugation at $14,300 \times g$ for $15 \mathrm{~min}$ at $4^{\circ} \mathrm{C}$. The 372 heat-treated spores were suspended in ice-cold germination buffer (50 mM Tris- $\mathrm{HCl}(\mathrm{pH} 7.4), 10 \mathrm{mM}$ $373 \mathrm{NaCl}$ and $100 \mathrm{mM} \mathrm{L}$-alanine) at an OD600 of 15 . The spore suspension (1.3 $\mu \mathrm{l})$ was dropped onto the 374 solid agarose pad, immediately covered by a cover slide $(18 \times 18 \mathrm{~mm})$ and was now ready for time375 lapse microscopy.

376 A Nikon Eclipse Ti-E microscope (Nikon Instruments, Tokyo, Japan) equipped with an sCmos camera 377 (Hamamatsu Flash 4.0 V2, Hamamatsu City, Japan), phase-contrast, and wide-field fluorescence 
components was used to track gemination of $B$. cereus spores for 4 hours with 10 min intervals. by four images, phase-contrast, SGFP2 fluorescence (excitation at $470 \mathrm{~nm}$ and emission at $516 \mathrm{~nm}$ ), mScarlet-I (excitation at $555 \mathrm{~nm}$ and emission at $593 \mathrm{~nm}$ ) and FRET (excitation at $470 \mathrm{~nm}$ and emission at $593 \mathrm{~nm}$ ). Spores of $B$. cereus strain 006 expressing GerR(A-C-B)-SGFP2 and spores of $B$. cereus strain 014 expressing SpoVAEa-SGFP2 were captured by phase-contrast and SGFP2 images. Spores of $B$. cereus 015 expressing SpoVAEa-SGFP2 and GerD-mScarlet-I were captured by phasecontrast, SGFP2 and mScarlet-I images. Spores of B. cereus 007 expressing GerD-mScarlet-I were captured by phase-contrast and mScarlet-I images.

All 16-bit type images taken in germination assays were converted to 32-bit type. Selection and measurement of the area of background in samples without an image were carried out, and background was subtracted by the tool of Process-Math-Subtract in Fiji/lmageJ. The germinated spores were analyzed and various intensities of individual spores measured using the ObjectJ (https://sils.fnwi.uva.nl/bcb/objectj/examples/sporetrackerc/MD/SporeTrackerC.html).

\section{Acknowledgments}

We acknowledge the Van Leeuwenhoek Center for Advanced Microscopy (LCAM) at the University of Amsterdam for offering the microscopy platform. We would like to thank Ronald M. P. Breedijk for his help in microscopy. We appreciate Juan Wen for sharing the raw data on the high frequency timelapse images of SpoVA-SGFP2 in one B. subtilis spore. Finally, Yan Wang acknowledges the China Scholarship Council for her PhD scholarship.

\section{References}

1. Ehling-Schulz, M.; Lereclus, D.; Koehler, T.M. The Bacillus cereus Group: Bacillus species with pathogenic potential. Microbiol. Spectr. 2019, 7, doi:10.1128/microbiolspec.GPP3-0032-2018.

2. Jessberger, N.; Dietrich, R.; Granum, P.E.; Märtlbauer, E. The Bacillus cereus food infection as multifactorial process. Toxins (Basel) 2020, 12, doi:10.3390/toxins12110701.

3. Jovanovic, J.; Ornelis, V.F.M.; Madder, A.; Rajkovic, A. Bacillus cereus food intoxication and toxicoinfection. Compr. Rev. Food Sci. Food Saf. 2021, 20, 3719-3761, doi:10.1111/1541-4337.12785.

4. McKenney, P.T.; Driks, A.; Eichenberger, P. The Bacillus subtilis endospore: assembly and functions of the multilayered coat. Nat. Rev. Microbiol. 2013, 11, 33-44, doi:10.1038/nrmicro2921.

5. Pelczar, P.L.; Setlow, P. Localization of the germination protein GerD to the inner membrane in Bacillus subtilis spores. J. Bacteriol. 2008, 190, 5635-5641, doi:10.1128/JB.00670-08.

6. Pelczar, P.L.; Igarashi, T.; Setlow, B.; Setlow, P. Role of GerD in germination of Bacillus subtilis spores. J. Bacteriol. 2007, 189, 1090-1098, doi:10.1128/JB.01606-06.

7. Wang, Y.; Breedijk, R.M.P.; Hink, M.A.; Bults, L.; Vischer, N.O.E.; Setlow, P.; Brul, S. Dynamics of germinosome formation and FRET-based analysis of interactions between GerD and germinant receptor subunits in Bacillus cereus spores. Int. J. Mol. Sci. 2021, 22, doi:10.3390/ijms222011230. 
415

8. Fort, P.; Errington, J. Nucleotide sequence and complementation analysis of a polycistronic sporulation operon, spoVA, in Bacillus subtilis. J. Gen. Microbiol. 1985, 131, 1091-1105, doi:10.1099/00221287-131-51091.

9. Perez-Valdespino, A.; Li, Y.; Setlow, B.; Ghosh, S.; Pan, D.; Korza, G.; Feeherry, F.E.; Doona, C.J.; Li, Y.Q.; Hao, B.; et al. Function of the SpoVAEa and SpoVAF proteins of Bacillus subtilis spores. J. Bacteriol. 2014, 196, 2077-2088, doi:10.1128/JB.01546-14.

10. Vepachedu, V.R.; Setlow, P. Analysis of interactions between nutrient germinant receptors and SpoVA proteins of Bacillus subtilis spores. FEMS Microbiol. Lett. 2007, 274, 42-47, doi:10.1111/j.15746968.2007.00807.x.

11. Wen, J.; Vischer, N.O.; de Vos, A.L.; Manders, E.M.M.; Setlow, P.; Brul, S. Organization and dynamics of the SpoVAEa protein, and its surrounding inner membrane lipids upon germination of Bacillus subtilis spores. bioRxiv 2021, doi:10.1101/2021.11.20.469378.

12. Moir, A.; Cooper, G. Spore germination. Microbiol. Spectr. 2015, 3, doi:10.1128/microbiolspec.TBS-00142012.

13. Shen, A.; Edwards, A.N.; Sarker, M.R.; Paredes-Sabja, D. Sporulation and germination in Clostridial pathogens. Microbiol. Spectr. 2019, 7, doi:10.1128/microbiolspec.GPP3-0017-2018.

14. Christie, G.; Setlow, P. Bacillus spore germination: Knowns, unknowns and what we need to learn. Cell. Signal. 2020, 74, 109729, doi:10.1016/j.cellsig.2020.109729.

15. Swerdlow, B.M.; Setlow, B.; Setlow, P. Levels of $\mathrm{H}+$ and other monovalent cations in dormant and germinating spores of Bacillus megaterium. J. Bacteriol. 1981, 148, 20-29, doi:10.1128/jb.148.1.2029.1981 .

16. Li, Y.; Davis, A.; Korza, G.; Zhang, P.; Li, Y.-Q.; Setlow, B.; Setlow, P.; Hao, B. Role of a SpoVA protein in dipicolinic acid uptake into developing spores of Bacillus subtilis. J. Bacteriol. 2012, 194, 1875-1884, doi:10.1128/JB.00062-12.

17. Vary, J.C.; Halvorson, H.O. Kinetics of germination of Bacillus spores. J. Bacteriol. 1965, 89, 1340-1347, doi:10.1128/jb.89.5.1340-1347.1965.

18. Hashimoto, T.; Frieben, W.R.; Conti, S.F. Microgermination of Bacillus cereus spores. J. Bacteriol. 1969, 100, 1385-1392, doi:10.1128/jb.100.3.1385-1392.1969.

19. Kong, L.; Zhang, P.; Wang, G.; Yu, J.; Setlow, P.; Li, Y.-Q. Characterization of bacterial spore germination using phase-contrast and fluorescence microscopy, Raman spectroscopy and optical tweezers. Nat. Protoc. 2011, 6, 625-639, doi:10.1038/nprot.2011.307.

20. Wang, Y.; Boer, R. de; Vischer, N.; van Haastrecht, P.; Setlow, P.; Brul, S. Visualization of germination proteins in putative Bacillus cereus germinosomes. Int. J. Mol. Sci. 2020, 21, doi:10.3390/ijms21155198.

21. Hornstra, L.M.; Vries, Y.P. de; Vos, W.M. de; Abee, T.; Wells-Bennik, M.H.J. gerR, a novel ger operon involved in L-alanine- and inosine-initiated germination of Bacillus cereus ATCC 14579. Appl. Environ. Microbiol. 2005, 71, 774-781, doi:10.1128/AEM.71.2.774-781.2005.

22. Bolte, S.; Cordelières, F.P. A guided tour into subcellular colocalization analysis in light microscopy. J. Microsc. 2006, 224, 213-232, doi:10.1111/j.1365-2818.2006.01706.x.

23. Adler, J.; Parmryd, I. Quantifying colocalization by correlation: the Pearson correlation coefficient is superior to the Mander's overlap coefficient. Cytometry A 2010, 77, 733-742, doi:10.1002/cyto.a.20896.

24. Cabrera-Martinez, R.-M.; Tovar-Rojo, F.; Vepachedu, V.R.; Setlow, P. Effects of overexpression of nutrient receptors on germination of spores of Bacillus subtilis. J. Bacteriol. 2003, 185, 2457-2464, doi:10.1128/JB.185.8.2457-2464.2003. 
25. Hornstra, L.M.; Vries, Y.P. de; Wells-Bennik, M.H.J.; Vos, W.M. de; Abee, T. Characterization of germination receptors of Bacillus cereus ATCC 14579. Appl. Environ. Microbiol. 2006, 72, 44-53, doi:10.1128/AEM.72.1.44-53.2006.

26. Zhang, J.; Griffiths, K.K.; Cowan, A.; Setlow, P.; Yu, J. Expression level of Bacillus subtilis germinant receptors determines the average rate but not the heterogeneity of spore germination. J. Bacteriol. 2013, 195, 1735-1740, doi:10.1128/JB.02212-12.

27. Artzi, L.; Alon, A.; Brock, K.P.; Green, A.G.; Tam, A.; Ramírez-Guadiana, F.H.; Marks, D.; Kruse, A.; Rudner, D.Z. Dormant spores sense amino acids through the $B$ subunits of their germination receptors. Nat. Commun. 2021, 12, 6842, doi:10.1038/s41467-021-27235-2.

28. Blinker, S.; Vreede, J.; Setlow, P.; Brul, S. Predicting the structure and dynamics of membrane protein GerAB from Bacillus subtilis. Int. J. Mol. Sci. 2021, 22, doi:10.3390/ijms22073793.

29. Pandey, R.; Beek, A. ter; Vischer, N.O.E.; Smelt, J.P.P.M.; Brul, S.; Manders, E.M.M. Live cell imaging of germination and outgrowth of individual Bacillus subtilis spores; the effect of heat stress quantitatively analyzed with SporeTracker. PLoS One 2013, 8, e58972, doi:10.1371/journal.pone.0058972. 